\title{
Algorithm of FBG Spectrum Distortion Correction for Optical Spectra Analyzers with CCD Elements
}

\author{
Vladimir Anfinogentov ${ }^{1}{ }^{(}, K_{\text {Kamil Karimov }}{ }^{1}$, Artem Kuznetsov ${ }^{1}{ }^{\circledR}$, Oleg G. Morozov ${ }^{1}{ }^{\oplus}$, Ilnur Nureev ${ }^{1}$, \\ Airat Sakhabutdinov ${ }^{1, *} \mathbb{1}$, Konstantin Lipatnikov ${ }^{1}$, Safaa M. R. H. Hussein ${ }^{2}$ and Mustafa H. Ali ${ }^{3}(\mathbb{C}$ \\ 1 Department of Radiophotonics and Microwave Technologies, Kazan National Research Technical University \\ Named after A.N. Tupolev-KAI, K. Marx Str. 10, 420111 Kazan, Russia; v.anfinogentov@yandex.ru (V.A.); \\ mail12kamil2000@mail.ru (K.K.); AAKuznetsov@kai.ru (A.K.); microoil@mail.ru (O.G.M.); \\ n2i2@mail.ru (I.N.); klipatnikov87@mail.ru (K.L.) \\ 2 Department of physics, College of Education for Pure Sciences, University of Karbala, Karbala 56001, Iraq; \\ safaa.mohammed@uokerbala.edu.iq \\ 3 College of Dentistry, University of Mustansiriyah, Baghdad 14022, Iraq; mustafa.h@uomustansiriyah.edu.iq \\ * Correspondence: azhsakhabutdinov@kai.ru
}

check for

updates

Citation: Anfinogentov, V.; Karimov, K.; Kuznetsov, A.; Morozov, O.G.;

Nureev, I.; Sakhabutdinov, A.;

Lipatnikov, K.; Hussein, S.M.R.H.; Ali, M.H. Algorithm of FBG Spectrum Distortion Correction for Optical Spectra Analyzers with CCD

Elements. Sensors 2021, 21, 2817.

https://doi.org/10.3390/s21082817

Academic Editor: Damien Kinet

Received: 24 February 2021

Accepted: 15 April 2021

Published: 16 April 2021

Publisher's Note: MDPI stays neutral with regard to jurisdictional claims in published maps and institutional affiliations.

Copyright: (c) 2021 by the authors. Licensee MDPI, Basel, Switzerland. This article is an open access article distributed under the terms and conditions of the Creative Commons Attribution (CC BY) license (https:/ / creativecommons.org/licenses/by/ $4.0 /)$.

\begin{abstract}
Nonlinear spectrum distortions are caused by the peculiarities of the operation of chargecoupled device elements (CCD), in which the signal exposition time (Time of INTegration-TINT) is one of the significant parameters. A change of TINT on a CCD leads to a nonlinear distortion of the resulting spectrum. A nonlinear distortion of the spectrum, in turn, leads to errors in determining the central wavelength of fiber Bragg gratings (FBGs) and spectrally sensitive sensors, which, in general, negatively affects the accuracy of the measuring systems. This paper proposes an algorithm for correcting the nonlinear distortions of the spectrum obtained on a spectrum analyzer using CCD as a receiver. It is shown that preliminary calibration of the optical spectrum analyzer with subsequent mathematical processing of the signal makes it possible to make corrections in the resulting spectrum, thereby leveling the errors caused by measurements at different TINT.
\end{abstract}

Keywords: nonlinear spectrum distortions; signal exposition time; fiber Bragg grating; fiber Bragg sensors; fiber optic sensors; fiber optic interrogator; optical spectrum analyzer; charge-coupled device elements; CCD

\section{Introduction}

It is widely known that in recent years, fiber-optic measuring systems have become increasingly relevant. In fiber-optic sensor systems, various technologies of interrogation and multiplexing are used [1-5]. Different technologies are applied for dividing fiber-optic spectral-sensitive sensors: by wavelength [1], by time response [2], by frequency [3], by polarization [4], and by spatial [5] multiplexing. To determine the average wavelength of the sensors, optical analyzers, such as spectrometers with tunable Fabry-Perot system interferometers, or diffraction gratings with CCD arrays, are used. The complexity of multiplexing technologies is also related to the fact that any spectrum overlaps of FBG spectra leads to significant errors in measurements of their central wavelengths [6-8]. The multiplexing technologies and microwave-photon interrogation methods of spectrally-encoded and addressed Bragg gratings have been developed; they allow separating, spectrally, the responses of sensors in the same frequency range [9-12]. Some researchers worked on sensor detection and tracking using Slepyan codes [13-16]; thus, a measurement of temperature and deformation in the case of sensors spectra overlapping became possible. Recently, a more convenient technology, based on addressable fiber Bragg structures, was proposed [17]. It made it possible to design distributed sensor systems with a large number of sensors without complicated optoelectronic schemes [17-19].

Despite significant progress in this direction, the classical optical spectrum analyzers, using diffraction gratings with CCD, have yet to develop their full potential. The attractive- 
ness is in the lower cost of such devices, compared to interrogators on tunable filters (with comparable measurement errors). The source of optical radiation is superluminescent laser diodes with a spectral width that overlaps the working spectral range of the analyzer.

The information parameter of the FBG sensor is the shift of its central wavelength. The data obtained from the CCD array allow measurements with an accuracy of $\sim 160 \mathrm{pm}$, which is not acceptable. Therefore, to improve the accuracy, an approximation is used. Various types of approximation have been investigated: Gaussian curve; second-order parabola using three upper points [20]; a parabola using the least-squares method; approximating the position of the central wavelength using the center of mass method; and others [21,22]. All investigated methods make it possible to determine the central wavelength FBG with a margin of error; however, the center of mass method gives the best accuracy.

\section{Problem Explanation}

For an experimental study of the FBG sensor interrogating system, its prototype was assembled. The IBSEN I-MON 512 USB as a spectrometer, a SLD-761-HP1-DIL as a broadband light source, and an FS62WSS (HBM) as a bore-type temperature sensor were used. The sensor response was measured at several constant temperature values maintained by a thermostat with an accuracy of $\pm 0.1^{\circ} \mathrm{C}$. The dependence "shift of the central wavelength-temperature" was plotted based on the measurement results. The second series of measurements were made at the facility, where the sensor was connected to the device through an existing long optical line. The decrease in the optical signal power was compensated by the increase in the exposition time (TINT) of the CCD array, which made it possible to "scale" the spectrum to the required signal-to-noise ratio. The spectrometer manufacturer (IBSEN) uses the term TINT as the variable and command in the software, and the term means "Time of INTegration". However, this approach led to the fact that the previously obtained correspondence "central wavelength-temperature shift" began to be violated: at the same temperature, the value of the central wavelength differed by tens of picometers (equivalent to an error of several degrees Celsius), from which a hypothesis was put forward about the nonlinear deformation of the spectrum with a change in the accumulation time.

It should be noted that there is no mention of this phenomenon in the user's manual of the spectrometer, which is, in our opinion, due to the fact that the manufacturer considers the spectrometer as an end product; in this regard, its use as an element of a more complex system is not within their goals.

In the works of other authors, it is shown that CCD-based spectrometers have a number of spectrum distortions, such as nonlinear pixel sensitivity to incident light [23] and chromatic distortions [24-26]. In these works, the sources of these errors are considered, as well as the methods of their compensation. In our work, we consider the spectrometer as a "black box", so we are not interested in the physical mechanisms of the spectrum distortion. We only operate with the data that it is capable of producing (taking into account the built-in mechanisms for picking up and converting the analog output of the CCD to the digital output) in response to standard commands-requests. It is also worth noting the work [27], where a modification of the algorithm for calculating the information characteristics of the FBG spectrum with its significant distortions is proposed.

Nevertheless, the interrogation system must consider the spectral characteristics both of spectrum analyzers and broadband light sources. It is necessary to calibrate spectrum analyzers and light sources jointly if we want to use their combination as an FBG interrogation system to avoid the FBG spectrum distortion due to different exposition times.

Thus, the task is to study the influence of the TINT parameter on distortions of the shape of the response spectrum of the FBG sensor, and advise methods to improve them.

\section{Collecting of Initial Data}

In order to solve this problem, a described experimental set-up was used. Here, the same fiber-optic sensor based on FBG was interrogated from two ends of the fiber. The 
optical line length connected to the fiber sensor at one end was equal to $10 \mathrm{~m}$, and at the other end was equal to $10 \mathrm{~km}$. The maximum amplitude of the spectral response from the sensor was set at $\sim 40,000$ quantization levels of the analog-to-digital converter to ensure an acceptable signal-to-noise ratio. This, in turn, led to the fact that the sensor was interrogated from the short end with TINT equal to $20 \mathrm{~ms}$, and from the long end equal to $135 \mathrm{~ms}$. The central wavelength was determined by the center of mass method for the same wavelength range of this sensor. It was found that the error in determining the central wavelength value of FBG in this experimental setup could reach $10 \mathrm{pm}$, which caused an error equal to $\sim 1 \mathrm{~K}$ in temperature determination. Figure 1 shows the spectral range of FBG, measured at three different values of TINT, the ordinate axis is shown in convenient units, normed at 40,000 quantization levels. The used spectrometer (Ibsen I-MON) has a built-in temperature sensor, the data from which are used to compensate the temperature fluctuations of the spectrometer elements (optical and electronic paths) and associated errors in the interpretation of the FBG spectrum. The method of this compensation is described in the documentation on Ibsen I-MON.

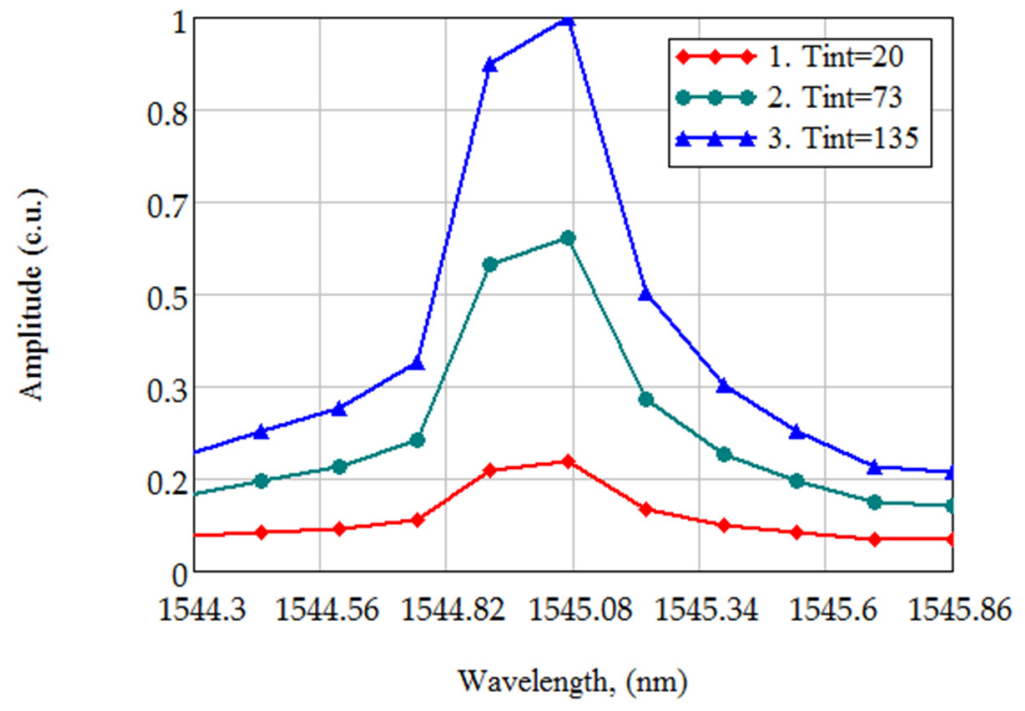

Figure 1. The FBG spectrum, measured at different TINT values (20, 73, $135 \mathrm{~ms})$.

The central wavelength value, calculated for the same spectrum of FBG at different TINT values, can differ significantly in practice. The difference in determining the FBG central wavelength depends on the chosen method and can reach $40 \mathrm{pm}$. For example, as shown in Figure 1, the differences between the central wavelength values for FBG at different values of TINT (20,73, and $135 \mathrm{~ms})$, are 7.6, 9.5, and $1.9 \mathrm{pm}$. This accuracy cannot be satisfactory, when the requirements for the temperature determining the accuracy is less than $1 \mathrm{~K}$.

For further investigation of the hypothesis, the initial broadband spectra were obtained at different values of TINT on the spectrum analyzer. Figure 2 shows the spectral characteristics of broadband laser radiation, obtained at different values of TINT (curve 1-20 ms, 2-48 ms, 3-77 ms, 4-106 ms, 5-135 ms).

For eleven different characteristic points of the spectrum, the dependence of their amplitudes on TINT was received; the dependences for these points with different initial values of the amplitude on TINT are shown in different colors in Figure 3. The dependence of the amplitude on TINT was plotted for the TINT range from 20 to 135 ms with the step equal to $1 \mathrm{~ms}$, which provided 116 measurements. 


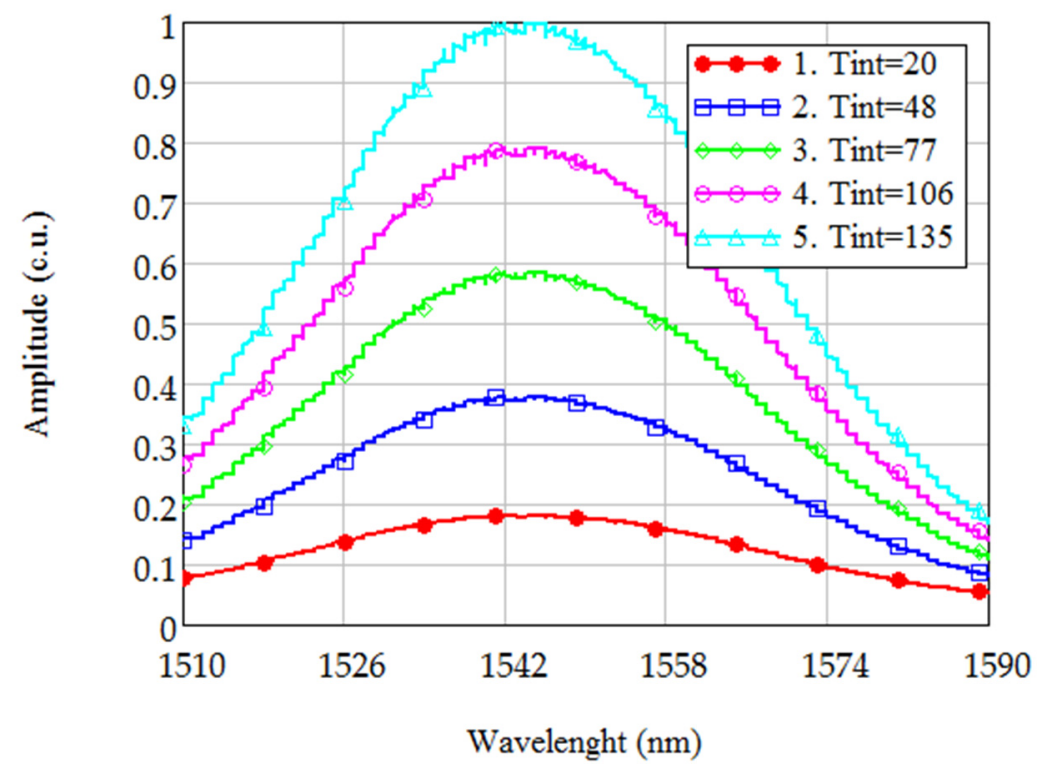

Figure 2. The broadband radiation spectra, obtained at different TINT values (1-20 ms, 2-48 ms, 3-77 ms, 4-106 ms, 5-135 ms).

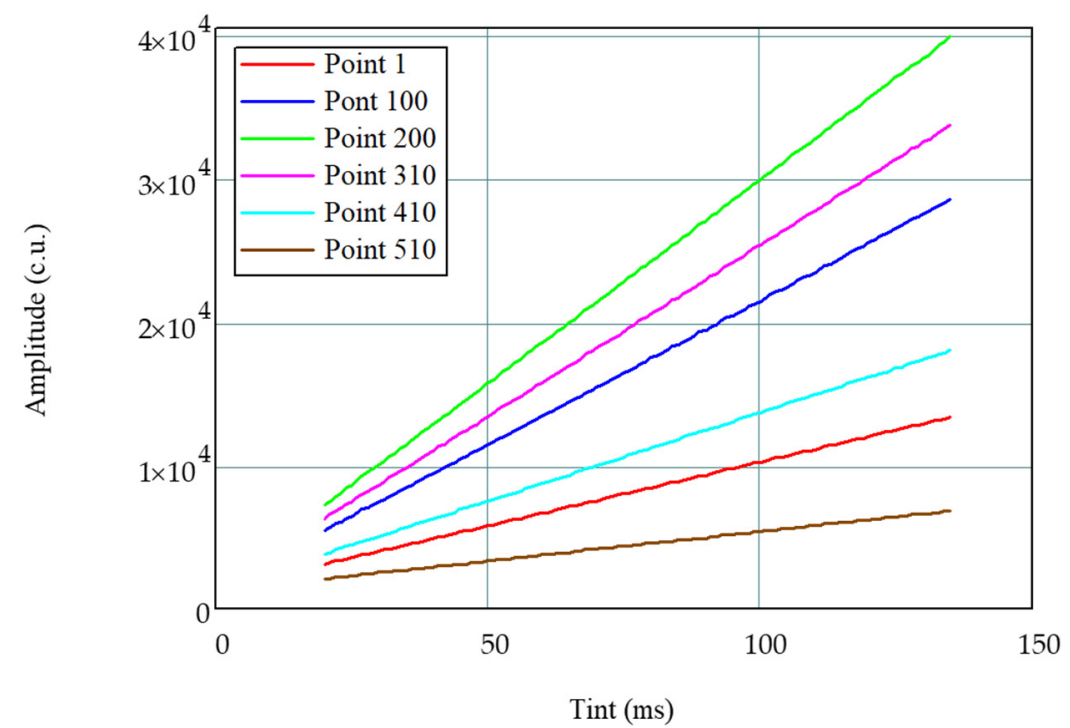

Figure 3. The dependence of the amplitude of different points of the spectrum on TINT.

From the dependences shown in Figure 3, it can be seen that, with an increase in TINT, a linear increase in the amplitude occurs; however, the coefficient of this linear dependence (the slope of the straight line) itself depends on the amplitude initial value, which is measured at the initial TINT value. In addition, it can be seen that the higher the amplitude, which is measured at the initial TINT value (at $20 \mathrm{~ms}$ ), the faster it grows with the TINT increase. This leads to nonlinear distortion of the spectrum and, ultimately, to errors in determining the FBG central wavelength.

The dependence of the slope angle on the initial value of the amplitude obtained at the initial value of TINT was received. This dependence is shown in Figure 4. As can be seen from Figure 4, the slope of the linear dependence has a linear dependence on the initial amplitude. 


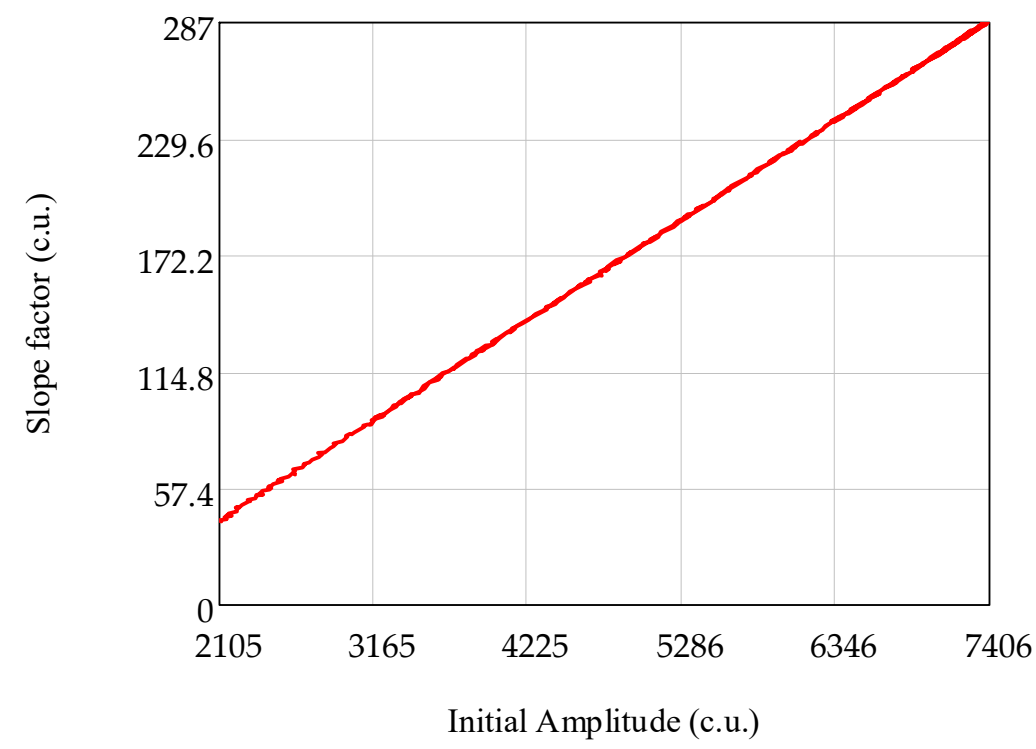

Figure 4. Dependence of the slope on the initial amplitude.

\section{Mathematical Processing}

The spectral response of the broadband source radiation for initial TINT value $\left(t_{0}\right)$ can be obtained:

$$
A_{i, 0}, i=\overline{1, N},
$$

where $N$ is the number of sampling points of the spectrum, $i=1$ and $i=N$ correspond to minimal and maximum wavelengths of range, respectively.

The amplitude increases linearly with TINT increasing, which allows approximating the dependence by a straight line:

$$
A(t)=A_{0}+k\left(A_{0}\right) \cdot\left(t-t_{0}\right),
$$

where $t_{0}$ is the initial TINT value, at which the calibration is performed; $t$ is the arbitrary TINT value; $k\left(A_{0}\right)$ is the linear dependence slope; and $A_{0}$ is the amplitude, obtained at the initial TINT $\left(t_{0}\right)$.

Based on the fact that the slope of the linear dependence (Figure 4) also increases linearly with the increasing amplitude, measured at the initial TINT, the slope of the linear dependence in Equation (2) can be represented as its linear dependence on the initial value of the amplitude, obtained at initial TINT. This statement can be formulated as follows:

$$
k\left(A_{0}\right)=\alpha \cdot A_{0}+\beta .
$$

Substituting Equation (3) into Equation (2), we obtain the dependence of the amplitude on TINT and the initial value of the amplitude, obtained at initial TINT value. A field of amplitude values depending on the TINT and the initial value of the amplitude, measured at initial TINT, can be obtained:

$$
A(t)=A_{0}+\left(\alpha \cdot A_{0}+\beta\right) \cdot\left(t-t_{0}\right),
$$

where $\alpha$ and $\beta$ are the coefficients of the linear dependence, $t_{0}$ is initial TINT, at which the amplitudes $A_{0}$ are measured.

It is possible to obtain a field of amplitude values depending on TINT and the initial value of the amplitude, measured at the initial TINT value, for the entire spectrum of the broadband radiation source:

$$
\left\{A_{i, j}, t_{j}\right\}, i=\overline{1, N}, j=\overline{0, M},
$$


where $N$ is the number of the spectrum sampling points, $M$ is the number of points for the TINT, changing in the range from $t_{0}$ to $t_{\mathrm{M}}$, namely $t_{j}=t_{0}+\mathrm{j}\left(t_{\mathrm{M}}-t_{0}\right) / M$.

Therefore, the measured amplitude $A_{i, j}\left(t_{j}, A_{i, 0}\right)$ for $i$-th sampling point can be written as dependence on TINT $\left(t_{j}\right)$ and on the initial amplitude value $A_{i, 0}$ for each:

$$
A_{i, j}\left(t_{j}, A_{i, 0}\right)=A_{i, 0}+\left(t_{j}-t_{0}\right)\left(\alpha \cdot A_{i, 0}+\beta\right), \quad i=\overline{1, N}, j=\overline{1, M} .
$$

The measured field of values Equation (5) makes it possible to determine the value of the slope of the linear dependence $k\left(A_{i, 0}\right)$ in Equation (2) for each initial value of the amplitude in each $i$-th spectrum point by calculating the characteristic points $\left\{A_{i, 0}, k\left(A_{i, 0}\right)\right\}$, $i=1, N$ using the least square method by formula:

$$
K_{i}=k\left(A_{i, 0}\right)=\sum_{j=1}^{M}\left(A_{j, i}-A_{0, i}\right)\left(t_{j}-t_{0}\right) / \sum_{j=1}^{N}\left(t_{j}-t_{0}\right)^{2}, \quad i=\overline{1, N} .
$$

It gives a set of values $\left\{A_{i, 0}, K_{i}\right\}, i=1, N$, which, in turn, allow determining the $\alpha$ and $\beta$ values-the linear dependence coefficients of the slope on $A_{0}$ in Equation (3), by the linear equations system solving the following:

$$
\left(\begin{array}{cc}
\sum_{i=1}^{N} A_{i, 0}^{2} & \sum_{i=1}^{N} A_{i, 0} \\
\sum_{i=1}^{N} A_{i, 0} & N
\end{array}\right) \cdot\left(\begin{array}{c}
\alpha \\
\beta
\end{array}\right)=\left(\begin{array}{c}
\sum_{i=1}^{N} A_{i, 0} K_{i} \\
\sum_{i=1}^{N} K_{i}
\end{array}\right) .
$$

Thus, after calculating the set of values $\left\{A_{i, 0}, K_{i}\right\}, i=1, N$, and the coefficients of the linear dependence of the slope in Equation (3)- $\alpha$ and $\beta$, in relation to Equation (6), the entire right-hand side becomes known. It allows to recalculate the initial amplitude of the arbitrary spectrum point, measured at initial TINT, depending on the amplitude, measured at arbitrary TINT $(t)$ :

$$
A_{i, 0}=\frac{A\left(t, A_{i, 0}\right)_{i}-\beta\left(t-t_{0}\right)}{1+\alpha \cdot\left(t-t_{0}\right)} .
$$

In Equation (9), $A\left(t, A_{i, 0}\right)$ is the amplitude, measured at arbitrary TINT, $\alpha$ and $\beta$ are the linear dependence coefficients of the slope in Equation (3), obtained as the solution of the linear equation system in Equation (8), and $A_{i, 0}$ is the amplitude, measured at initial TINT.

Thus, knowing the current value of the amplitude at a point in the spectrum, measured at an arbitrary TINT, providing a comfortable signal-to-noise ratio, allows determining the amplitude value, measured at initial TINT, which is used in the calibration process for this sensor.

\section{Experimental Setup}

The experimental setup is shown in Figure 5a. The experimental setup consists of the thermostat, interrogator (Figure $5 b$ ), fiber sensors under calibration, and controlling system. The thermostat is shown at the left side of Figure 5a; it has its own temperature sensor which is connected to the interrogator by the RS-232 interface. The sensors are placed in the thermostat and held at a constant temperature. The thermostat's temperature is varied in the range with the given step. The interrogator is placed on the right side of the table in Figure 5a. The experimental setup is assembled so the sensors are affected only by temperature. 


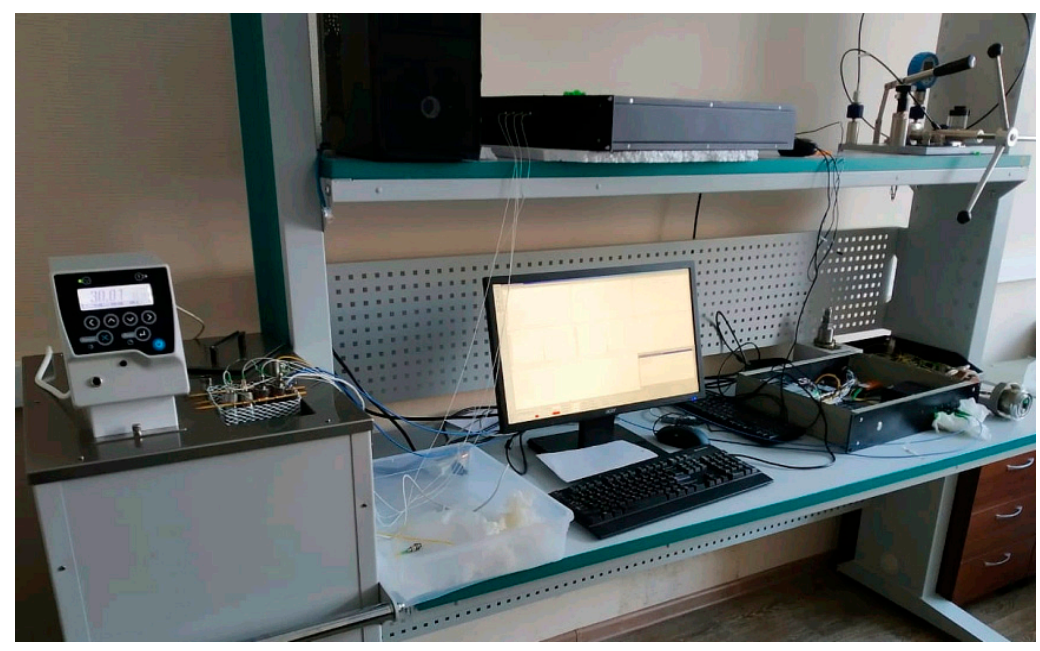

(a)

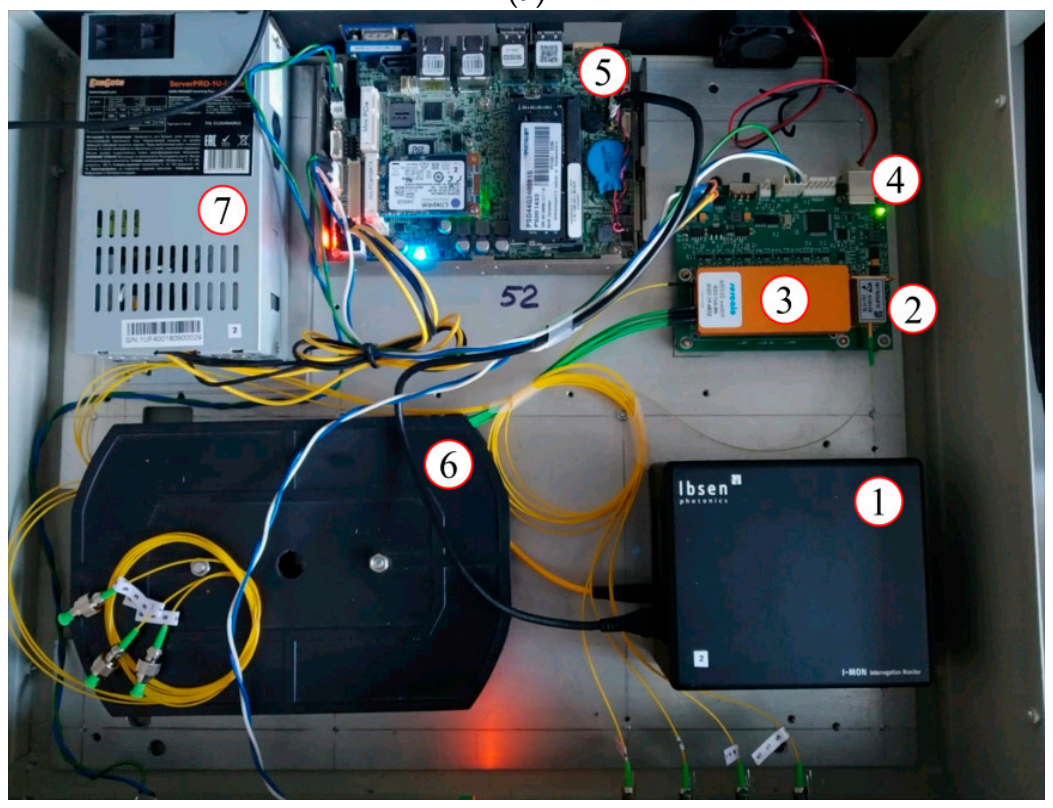

(b)

Figure 5. Experimental setup (a); FBG interrogator (b): 1-IBSEN I-MON 512 USB; 2-laser source SLD-761-HP1-DIL; 3-optic channel switch Sercalo MEMS switch rSC $1 \times 8$; 4-laser source and optical switch maintaining module; 5-computer Wafer ULT-3; 6-optical cross; 7-power supply.

The FBG interrogator (Figure 5b) includes the optical spectrum analyzer IBSEN IMON 512 with a USB interface-1. The laser source SLD-761-HP1-DIL-2 is used as the broadband optical light source. The optical channel switch Sercalo MEMS switch rSC $1 \times 8-3$ is used to switch channels to have a possibility to interrogate several optical channels consistently with the given interval $(\sim 100 \div 200 \mathrm{~ms})$. In this interrogator model, we used eight independent channels with eight sensors in each channel. The optical light source with the channel switch is maintained by our own designed control board-4. All measured data are collected on the on-board computer, Wafer ULT-3-5. The optical cross -6 is used for internal optical cabling. The common power supply-7 is used.

\section{Calibration Data}

The calibration curves received without and with the spectrum calibration algorithm are presented in Figure 6. All measurements were made for the bore-type temperature sensor FS62WSS (HBM) from two ends. The measurements made from the first end are marked by red rhombuses, and from the second end are marked by blue rhombuses. All 
measurements were made in the temperature range from 15 to $90{ }^{\circ} \mathrm{C}$ with a discrete step of $5{ }^{\circ} \mathrm{C}$. Fifty independent measurements of the FBG central wavelength were made in each temperature point; thus, each rhombus in the figure means one measurement.

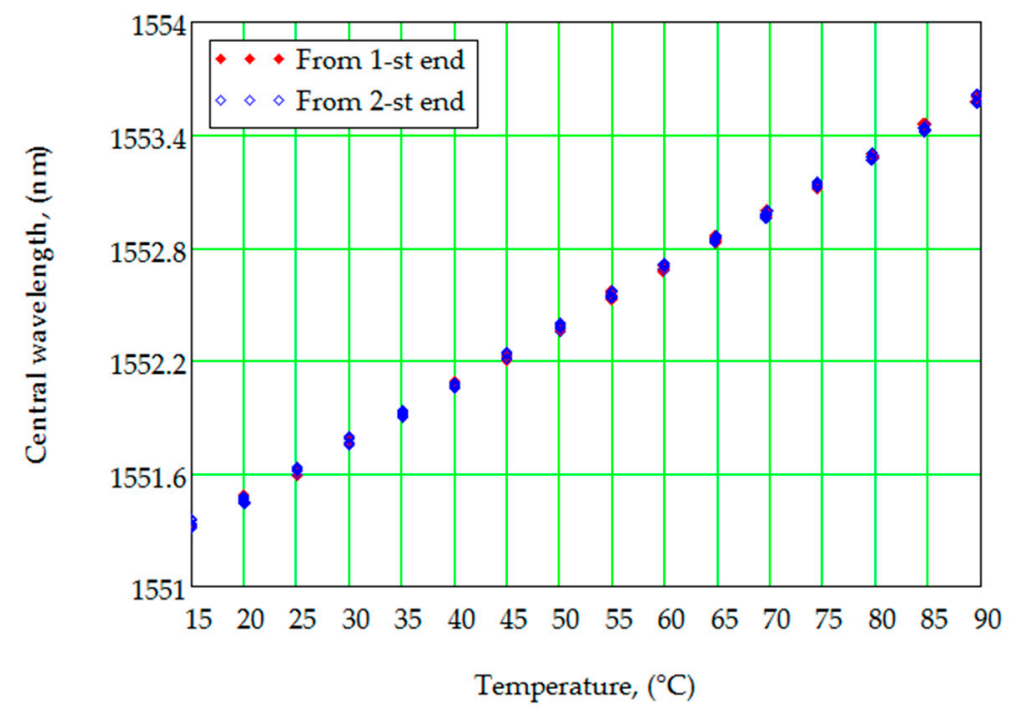

(a)

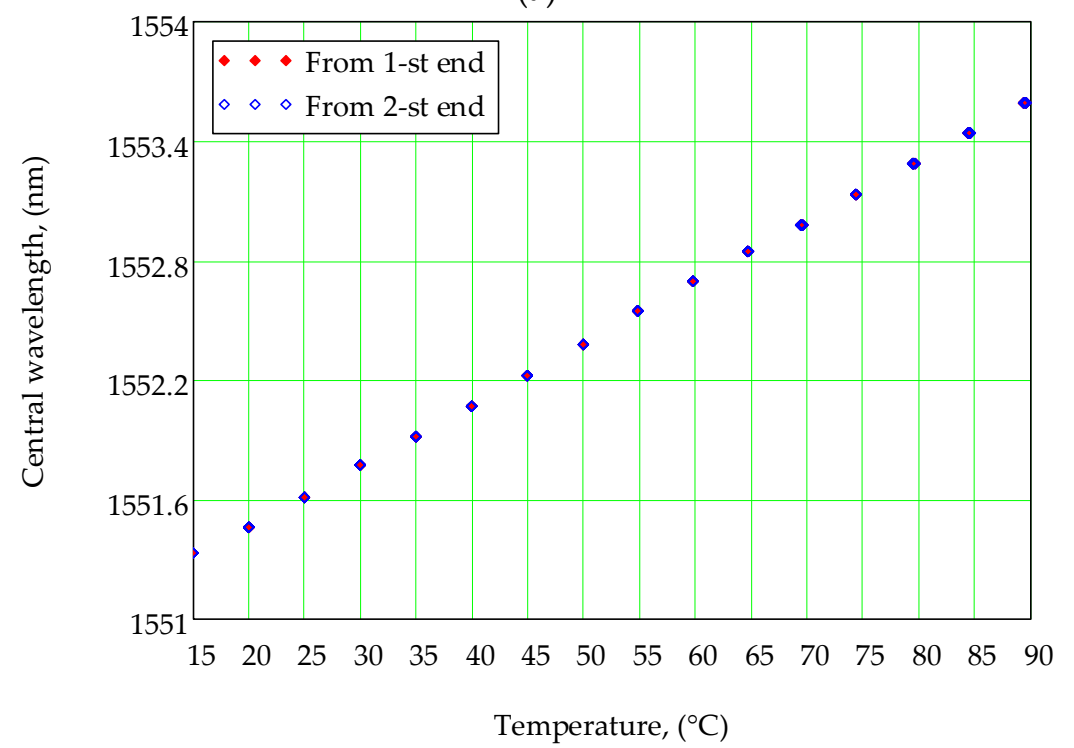

(b)

Figure 6. Calibration curves-FBG central wavelength dependences on temperature. The measured data: (a) without spectrum correction; (b) with spectrum correction.

As one can see, there is dispersion in obtaining the FBG central wavelength in the case when the spectrum calibration algorithm is not used (Figure 6a). The average error of the central wavelength approximation is $50 \mathrm{pm}$, which is equivalent to $5 \mathrm{~K}$. While the calibration dependence received with the spectrum correction algorithm gives an error approximation of the FBG central wavelength of less than $0.1 \mathrm{pm}$, that allows approximating the temperature with $0.01 \mathrm{~K}$ accuracy (Figure $5 \mathrm{~b}$ ).

The received results approve the necessity of using the preliminary mandatory calibration of the light source with the CCD element and using these calibration results to correct the FBG spectra from the spectrum analyzer. 


\section{Conclusions}

The proposed algorithm allows excluding nonlinear distortions of the fiber Bragg grating spectrum response, caused by different signal integration times on charge-coupled devices.

The spectrum correction, performed according to the proposed algorithm, allows excluding limitations associated with the fact that the calibration of all sensors in the fiber-optic measurement system, based on CCD, must be carried out at the same signal integration time values, which will be used in exploitation.

As a result, the compensation of nonlinear distortions allows calibrating sensors independently from the measuring system. In addition, it removes restrictions on strictly fixed optical lengths from an interrogator to the sensor. Moreover, it gives the opportunity to simplify the array of sensors formed in an optical channel, and to replace sensors with one another arbitrarily during exploitation.

We are free to discuss this problem and our results with scientists and manufacturers.

Author Contributions: Conceptualization, V.A., O.G.M. and A.S.; methodology, A.S. and V.A.; software, K.K. and K.L.; validation, V.A., K.L., I.N., A.S. and O.G.M.; formal analysis, A.S., K.L., M.H.A., and S.M.R.H.H.; investigation, I.N., V.A., A.S., M.H.A., and K.K.; resources, A.K.; data curation, A.K., K.L., S.M.R.H.H., and M.H.A.; writing-original draft preparation, A.S. and A.K.; writing-review and editing, A.K. and A.S.; visualization, K.K. and K.L.; supervision, O.G.M.; project administration, O.G.M.; funding acquisition, A.K. and O.G.M. All authors have read and agreed to the published version of the manuscript.

Funding: A.S. and O.G.M. were funded by the Ministry of Science and Higher Education of the Russian Federation (Agreement No. 075-03-2020-051, Topic No. fzsu-2020-0020), A.K. was funded by a grant from the President of the Russian Federation for the state support of young Russian scientists—candidates of sciences MK-3421.2019.8 (Agreement No. 075-15-2019-309).

Institutional Review Board Statement: Not applicable.

Informed Consent Statement: Not applicable.

Data Availability Statement: The data presented in this study are available on request from the corresponding author. The data are not publicly available due to rules of our contract conditions with our customer.

Conflicts of Interest: The authors declare no conflict of interest.

\section{References}

1. Measures, R.M.; Melle, S.; Liu, K. Wavelength demodulated Bragg grating fiber optic sensing systems for addressing smart structure critical issues. Smart Mater. Struct. 1992, 1, 36-44. [CrossRef]

2. Davis, M.A.; Bellemore, D.G.; Kersey, A.D. Structural strain mapping using a wavelength/time division addressed fiber Bragg grating array. Smart Struct. Mater. Second Eur. Conf. 1994, 2361, 342-346. [CrossRef]

3. Matveenko, V.; Shardakov, I.; Voronkov, A.; Kosheleva, N.; Lobanov, D.; Serovaev, G.; Spaskova, E.; Shipunov, G. Measurement of strains by optical fiber Bragg grating sensors embedded into polymer composite material. Struct. Control. Health Monit. 2017, 25, e2118. [CrossRef]

4. Qiao, X.; Shao, Z.; Bao, W.; Rong, Q. Fiber Bragg Grating Sensors for the Oil Industry. Sensors 2017, 17, 429. [CrossRef] [PubMed]

5. Ma, Z.; Chen, X. Fiber Bragg gratings sensors for aircraft wing shape measurement: Recent applications and technical analysis. Sensors 2019, 19, 1-25. [CrossRef] [PubMed]

6. Karim, F. Full Matlab Code for Synthesis and Optimization of Bragg Gratings; Cambridge Scholars Publishing: Newcastle upon Tyne, UK, 2019; p. 24.

7. Cormier, G.; Boudreau, R.; Thériault, S. Real-coded genetic algorithm for Bragg grating parameter synthesis. J. Opt. Soc. Am. B 2001, 18, 1771-1776. [CrossRef]

8. Li, K. Review of the Strain Modulation Methods Used in Fiber Bragg Grating Sensors. J. Sensors 2016, 2016, 1-8. [CrossRef]

9. Koo, K.; Leblanc, M.; Tsai, T.; Vohra, S. Fiber-chirped grating Fabry-Perot sensor with multiple-wavelength-addressable freespectral ranges. IEEE Photon. Technol. Lett. 1998, 10, 1006-1008. [CrossRef]

10. Wei, Z.; Ghafouri-Shiraz, H.; Shalaby, H. New code families for fiber-Bragg-grating-based spectral-amplitude-coding optical CDMA systems. IEEE Photon. Technol. Lett. 2001, 13, 890-892. [CrossRef]

11. Dai, B.; Gao, Z.; Wang, X.; Kataoka, N.; Wada, N. Performance comparison of $0 / \pi$ - and $\pm \pi / 2$-phase-shifted superstructured Fiber Bragg grating en/decoder. Opt. Express 2011, 19, 12248-12260. [CrossRef] 
12. Triana, C.A.; Pastor, D.; Varon, M. Optical code division multiplexing in the design of encoded fiber Bragg grating sensors. Opt. Pura Apl. 2016, 49, 17-28. [CrossRef]

13. Triana, A.; Pastor, D. Interrogation of super-structured FBG sensors based on discrete prolate spheroidal sequences. Opt. Sens. 2017, 10231, 102310. [CrossRef]

14. Djordjevic, I.B.; Saleh, A.H.; Küppers, F. Design of DPSS based fiber bragg gratings and their application in all-optical encryption, OCDMA, optical steganography, and orthogonal-division multiplexing. Opt. Express 2014, 22, 10882-10897. [CrossRef]

15. Kim, Y.; Jeon, S.-W.; Kwon, W.-B.; Park, C.-S. A Wide Dynamics and Fast Scan Interrogating Method for a Fiber Bragg Grating Sensor Network Implemented Using Code Division Multiple Access. Sensors 2012, 12, 5888-5895. [CrossRef]

16. Triana, A.; Pastor, D.; Varón, M. A Code Division Design Strategy for Multiplexing Fiber Bragg Grating Sensing Networks. Sensors 2017, 17, 2508. [CrossRef]

17. Morozov, O.; Sakhabutdinov, A.; Anfinogentov, V.; Misbakhov, R.; Kuznetsov, A.; Agliullin, T. Multi-Addressed Fiber Bragg Structures for Microwave-Photonic Sensor Systems. Sensors 2020, 20, 2693. [CrossRef]

18. Agliullin, T.; Gubaidullin, R.; Sakhabutdinov, A.; Morozov, O.; Kuznetsov, A.; Ivanov, V. Addressed Fiber Bragg Structures in Load-Sensing Wheel Hub Bearings. Sensors 2020, 20, 6191. [CrossRef]

19. Gubaidullin, R.; Agliullin, T.; Ivanov, V.; Sakhabutdinov, A. Tire dynamic monitoring setup based on microwave photonic sensors. Opt. Technol. Telecommun. 2019, 11146, 111461J. [CrossRef]

20. Jiang, J.; Liu, T.; Liu, K.; Zhang, Y. Investigation of peak wavelength detection of fiber Bragg grating with sparse spectral data. Opt. Eng. 2012, 51, 034403. [CrossRef]

21. Morozov, O.G.; Kuznetsov, A.A.; Morozov, G.A.; Nureev, I.I.; Sakhabutdinov, A.Z.; Faskhutdinov, L.M.; Artemev, V.I. Smart Photonic Carbon Brush. In Proceedings of the International Society for Optical Engineering, Ufa, Russia, 26 March 2016; Sultanov, A.H., Morozov, O.G., Burdin, V.A., Bourdine, A.V., Andreev, V.A., Eds.; SPIE: Ufa, Russia, 2016; Volume 9807, p. 98070M. [CrossRef]

22. Gubaidullin, R.R.; Agliullin, T.A.; Ivanov, V.; Morozov, O.G.; Sakhabutdinov, A.Z. Tire Dynamic Monitoring Setup Based on Microwave Photonic Sensors. In Proceedings of the Optical Technologies for Telecommunications 2018, Ufa, Russia, 24 June 2019; Volume 11146, p. 111461J. [CrossRef]

23. Xia, G.; Liu, Q.; Zhou, H.; Yu, F. A non-linearity correction method of charge-coupled device array spectrometer. In Proceedings of the AOPC 2015: Optical Test, Measurement, and Equipment, Beijing, China, 1 October 2015; Volume 9677, p. 96770J. [CrossRef]

24. Fu, H.-K.; Liu, Y.-L.; Chen, T.-T.; Wang, C.-P.; Chou, P.-T. The Study of Spectral Correction Algorithm of Charge-Coupled Device Array Spectrometer. IEEE Trans. Electron Devices 2014, 61, 3796-3802. [CrossRef]

25. Czech, E.; Fryc, I. Spectral correction of the measurement CCD array. Opt. Eng. 2002, 41, 2402-2406. [CrossRef]

26. Sun, Y.C.; Huang, C.; Xia, G.; Jin, S.Q.; Lu, H.B. Accurate wavelength calibration method for compact CCD spectrometer. J. Opt. Soc. Am. A 2017, 34, 498-505. [CrossRef]

27. Lamberti, A.; Vanlanduit, S.; De Pauw, B.; Berghmans, F. Influence of Fiber Bragg Grating Spectrum Degradation on the Performance of Sensor Interrogation Algorithms. Sensors 2014, 14, 24258-24277. [CrossRef] 